\title{
Papers
}

\section{Stable partnership and progression to AIDS or death in HIV infected patients receiving highly active antiretroviral therapy: Swiss HIV cohort study}

Jim Young, Sabina De Geest, Rebecca Spirig, Markus Flepp, Martin Rickenbach, Hansjakob Furrer, Enos Bernasconi, Bernard Hirschel, Amalio Telenti, Pietro Vernazza, Manuel Battegay, Heiner C Bucher, Swiss HIV Cohort Study Group

\begin{abstract}
Objectives To explore the association between a stable partnership and clinical outcome in HIV infected patients receiving highly active antiretroviral therapy (HAART).

Design Prospective cohort study of adults with HIV (Swiss HIV cohort study).

Setting Seven outpatient clinics throughout Switzerland. Participants The 3736 patients in the cohort who started HAART before 2002 (median age 36 years, 29\% female, median follow up 3.6 years).

Main outcome measures Time to AIDS or death (primary endpoint), death alone, increases in CD4 cell count of at least 50 and 100 above baseline, optimal viral suppression (a viral load below 400 copies $/ \mathrm{ml}$ ), and viral rebound.

Results During follow up 2985 (80\%) participants reported a stable partnership on at least one occasion. When starting HAART, 52\% (545/1042) of participants reported a stable partnership; after five years of follow up 46\% (190/412) of participants reported a stable partnership. In an analysis stratified by previous antiretroviral therapy and clinical stage when starting HAART (US Centers for Disease Control and Prevention group A, B, or C), the adjusted hazard ratio for progression to AIDS or death was 0.79 (95\% confidence interval 0.63 to 0.98 ) for participants with a stable partnership compared with those without. Adjusted hazards ratios for other endpoints were 0.59 ( 0.44 to 0.79 ) for progression to death, 1.15 (1.06 to 1.24) for an increase in CD4 cells of 100 counts $/ \mu 1$ or more, and 1.06 (0.98 to 1.14) for optimal viral suppression. Conclusions A stable partnership is associated with a slower rate of progression to AIDS or death in HIV infected patients receiving HAART.
\end{abstract}

\section{Introduction}

Cohort studies have shown that social relationships influence mortality. Having fewer close friends or relatives, less frequent contact with other people, and reduced social participation have all been associated with increased mortality, especially in elderly people and in men. ${ }^{1-4}$ People living alone have a higher mortality from cardiovascular disease and a poorer prognosis after a cardiac event. ${ }^{56}$ However, little is known about the impact of social relationships on outcome for people infected with HIV, most of whom are under 40 years old.
Social support, the emotional or tangible support available from other people, is one of the primary ways in which social relationships influence health. ${ }^{7}$ In particular, greater emotional support is strongly associated with lower mortality. ${ }^{8}$ Social support may have physiological effects on the neuroendocrine or immune systems. ${ }^{9}{ }^{10}$ Poor social support has been linked to a more rapid decrease in CD4 cell counts in HIV infection. ${ }^{11}{ }^{12}$ Social support may have psychological effects, such as reducing anxiety and depression. ${ }^{13}$ Social support may also moderate risk behaviours and improve adherence to treatment. ${ }^{14} 15$

Little is known about the effect of social support on outcome in HIV infection. Most definitions of social support emphasise family ties or social activities, ${ }^{16}$ and these may be less relevant for people living with HIV. ${ }^{17}$ For many people with HIV, a partner may be the most important source of emotional and tangible support. We investigated the association between a stable partnership and disease progression in HIV infected people receiving highly active antiretroviral therapy (HAART).

\section{Methods}

Participants

The Swiss HIV cohort study is a prospective cohort study with continuing enrolment of HIV infected patients aged 16 years or over. All participants have given written informed consent. Since 1993, patients enrolled have been asked at visits scheduled every six months whether they have had sexual intercourse with a stable partner during the previous six months. From April 2000 on, this single question was asked as two separate questions (presence of stable partner and sexual intercourse with that partner) as part of a new questionnaire on sexual behaviour.

HAART became available to all residents of Switzerland in August 1996, when its cost was covered by compulsory health insurance. In this analysis, HAART is defined as the combination of at least two reverse transcriptase inhibitors with one boosted or non-boosted protease inhibitor or one non-nucleoside reverse transcriptase inhibitor. As of 28 February 2002, 5350 patients in the cohort had started HAART. We included in our analysis all patients who started HAART and had CD4 lymphocyte counts and plasma HIV RNA (viral load) measured within three months before starting HAART and at least one follow up visit more than one month after starting HAART. 


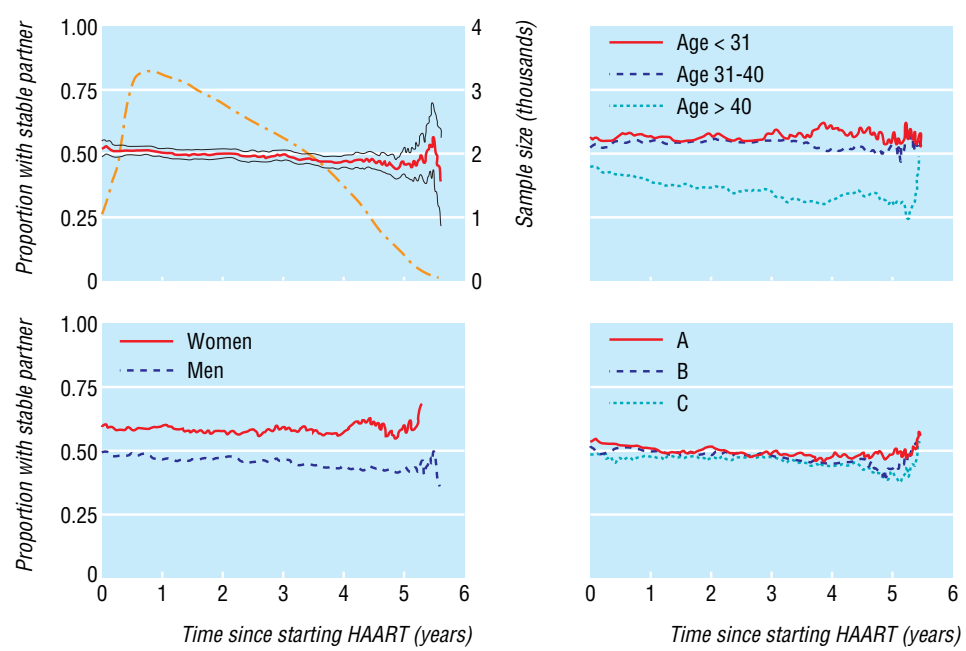

Fig 1 Proportion of participants reporting a stable partnership after starting highly active antiretroviral therapy (HAART): overall proportion (with 95\% confidence interval) and sample size (dotted line) (top left); proportion for each sex (bottom left); proportion for each of three age groups (top right); and proportion for each clinical stage at baseline (bottom right)

\section{Endpoints}

We considered the association between stable partnership and several clinical and surrogate marker endpoints. We selected two clinical endpoints: (a) new US Centers for Disease Control and Prevention (CDC) group C disease ${ }^{18}$ or death (primary endpoint) and $(b)$ death alone. We also considered four surrogate marker endpoints: $(a)$ increase in CD4 cell count of at least 50 above baseline, (b) increase in CD4 cell count of at least 100 above baseline, $(c)$ optimal viral suppression (a viral load below 400 copies $/ \mathrm{ml}$ ), and (d) viral rebound (the first of two consecutive measurements of more than 400 copies $/ \mathrm{ml}$ ) in patients achieving optimal viral suppression.

\section{Statistical analysis}

We estimated the proportion of patients in the cohort reporting a stable partnership since starting HAART. We included patients from their first follow up visit after starting HAART until their latest follow up visit. We assumed that patients reporting a stable partnership remained in a stable partnership until at a subsequent visit they either reported no stable partner or refused to answer the relevant questions. We defined stable partnership as reporting both a stable partner and sexual intercourse with that partner during the previous six months, so as to use a constant definition before and after April 2000.

We used Cox proportional hazards models to estimate the association between a stable partnership and disease progression since starting HAART. Our models included left truncated observations, strata and covariates, and a time dependent treatment variable. Firstly, some patients were registered with the cohort after starting HAART. We included these participants as left truncated observations so that they contributed to estimates only after registration. Secondly, we needed strata within which participants had roughly identical rates of disease progression. We stratified by previous antiretroviral therapy and by clinical stage at baseline (CDC group A, B, or C). This stratification by disease severity at baseline also avoids potential bias if sicker patients are less likely to form stable partnerships because of the severity of their illness. ${ }^{19}$ Thirdly, we needed to estimate the association between stable partnership and disease progression after adjustment for other variables that might potentially influence the rate of disease progression. We used as covariates CD4 cell counts and viral load at baseline, age and sex, and education and transmission group categories. Fourthly, we represented stable partnership by a time dependent variable to avoid "survivor treatment selection bias, ${ }^{19}$ whereby participants who had been followed for longer had had more opportunity to form a stable partnership. In our models "treatment" starts only when a patient first reports a stable partnership. Under the intention to treat principle, treatment then continues for a patient even if the stable partnership ends. This avoids potential bias caused by sicker patients discontinuing a stable partnership because of the severity of their illness.

We used SAS version 8.2 for the analyses. We assessed the evidence for an association between disease progression and another variable by using a hypothesis test based on the difference in the log likelihood ratio between the full model and a reduced model without that variable. We assessed the nature of any association between disease progression and another variable by using a hazard ratio. We give Wald based 95\% confidence intervals for all hazard ratios.

\section{Results}

\section{Participants}

As of 28 February 2002, 5350 patients in the cohort had started HAART. We analysed data from the $3736(70 \%)$ patients who had had CD4 cell counts (4130, 77\%) and viral load (4041, 76\%) measured within the three months before starting HAART and at least one follow up visit more than one month after starting HAART $(5105,95 \%)$. We followed these patients for a total of 12173 patient years (median 3.6 years per patient).

Patients included in our analysis were on average similar in age, sex, and most likely source of infection (transmission group) to those excluded (table 1). However, those included were more likely to have a higher level of education (more than mandatory schooling), less likely to belong to ethnic groups other than white European, less likely to be in CDC group C, and more likely to have had antiretroviral therapy before starting HAART.

When starting HAART, 52\% $(545 / 1042)$ of participants reported a stable partnership. This percentage decreased to around 46\% (190/412) after five years of follow up (fig 1a). Beyond five years, estimates of the percentage reporting a stable 
Table 1 Baseline characteristics for participants in the Swiss HIV cohort study on HAART and those in the analysis who did not reach the primary endpoint. Values are numbers (percentages) unless stated otherwise

\begin{tabular}{|c|c|c|c|c|}
\hline \multirow[b]{2}{*}{ Characteristic } & \multicolumn{2}{|c|}{ Cohort members on HAART } & \multicolumn{2}{|c|}{ Did not reach primary endpoint } \\
\hline & Included in analysis ( $\mathrm{n}=3736$ ) & Excluded $(n=1614)$ & Attended follow up* $(\mathrm{n}=2896)$ & Not followed up $(n=413)$ \\
\hline Median viral load ( $\log _{10}$ copies/ml) & 4.50 & - & 4.45 & 4.47 \\
\hline Median CD4 cell count (cells/ul) & 210 & - & 222 & 252 \\
\hline Median age (years) & 36 & 36 & 36 & 35 \\
\hline \multicolumn{5}{|l|}{ Sex: } \\
\hline Female & $1096(29)$ & $474(29)$ & $864(30)$ & $123(30)$ \\
\hline Male & $2640(71)$ & $1140(71)$ & $2032(70)$ & $290(70)$ \\
\hline \multicolumn{5}{|l|}{ Transmission group: } \\
\hline Homosexual & $1295(35)$ & $538(33)$ & $1076(37)$ & $87(21)$ \\
\hline Intravenous drug user & $1077(29)$ & $464(29)$ & $724(25)$ & $186(45)$ \\
\hline Heterosexual, othert, or unknown & $1364(37)$ & $612(38)$ & $1096(38)$ & $140(34)$ \\
\hline \multicolumn{5}{|l|}{ Education: } \\
\hline Higher & $2532(68)$ & $1026(64)$ & $2003(69)$ & $245(59)$ \\
\hline Basic or unknown & $1204(32)$ & $588(36)$ & $893(31)$ & $168(41)$ \\
\hline \multicolumn{5}{|l|}{ Ethnicity: } \\
\hline Other than white European & $392(10)$ & $214(13)$ & $315(11)$ & $50(12)$ \\
\hline White European or unknown & $3340(90)$ & $1400(87)$ & 2581 (89) & $363(88)$ \\
\hline \multicolumn{5}{|l|}{ Clinical stage: } \\
\hline A & $1670(45)$ & $718(44)$ & $1374(47)$ & $189(46)$ \\
\hline $\mathrm{B}$ & $1173(31)$ & $426(26)$ & $891(31)$ & $130(31)$ \\
\hline $\mathrm{C}$ & $893(24)$ & $470(29)$ & $631(22)$ & $94(23)$ \\
\hline \multicolumn{5}{|l|}{ Previous antiretroviral therapy: } \\
\hline No & $1912(51)$ & $890(55)$ & $1522(53)$ & $203(49)$ \\
\hline Yes & 1824 (49) & $724(45)$ & $1374(47)$ & $210(51)$ \\
\hline If yes, median duration (years) & 1.7 & 1.6 & 1.7 & 1.7 \\
\hline
\end{tabular}

HAART=highly active antiretroviral therapy.

*During the 12 months before 28 February 2002.

†Transmission through blood products or perinatal transmission.

Table 2 Number (percentage) of participants in each stratum reporting stable partnership at least once

Previous antiretroviral therapy

$\begin{array}{lll}\text { Yes } & \text { No } & \text { Total }\end{array}$

\begin{tabular}{|c|c|c|c|}
\hline \multicolumn{4}{|c|}{ Clinical stage: } \\
\hline A & $484 / 565$ (86) & $845 / 1105$ (76) & $1329 / 1670(80)$ \\
\hline$B$ & $633 / 748$ (85) & $321 / 425$ (76) & $954 / 1173(81)$ \\
\hline C & 416/511 (81) & $286 / 382$ (75) & $702 / 893 \quad(79)$ \\
\hline Total & $1533 / 1824$ (84) & $1452 / 1912$ (76) & $2985 / 3736 \quad(80)$ \\
\hline
\end{tabular}

partnership are unstable because they are based on a small sample; estimates based on a sample size of less than 30 are not shown. The decrease in the percentage reporting a stable partnership over time seems to be related to increasing age. The decrease was obvious among participants aged over 40 (fig 1c). The decrease in the percentage reporting a stable partnership was similar among participants in each of the three clinical stages at baseline (fig 1d). If infection with HIV made it more difficult to maintain a stable partnership, we might expect to see a more rapid decrease among sicker patients.

Of the 3736 patients in our analysis, 2985 (80\%) reported a stable partnership at least once during follow up. Those who had had antiretroviral therapy before starting HAART were likely to have registered earlier with the cohort and therefore had more time in which to form stable partnerships (table 2). Among the participants who had had previous antiretroviral therapy, the percentage reporting a stable partnership at least once was slightly higher for those at clinical stage A $(484 / 565,86 \%)$ than for those at stage C $(416 / 511,81 \%)$. This may reflect a higher mortality among patients who were sicker when starting HAART.

Of the $3432(92 \%)$ participants who achieved optimal viral suppression (RNA < 400 copies/ml) at least once, $3360(98 \%)$ had a CD4 cell count measured within the three months before they first achieved optimal suppression. We used these 3360 participants to estimate the association between stable partnership and the rate of progression to viral rebound.

\section{Primary endpoint}

Kaplan-Meier curves show that the initial rate of new disease or death was higher for patients starting HAART without previous antiretroviral therapy (fig 2). This is probably a result of immune restoration diseases (new disease within 90 days of starting HAART without previous antiretroviral therapy) rather than HIV progression. $.^{20} \log -\log$ survival curves (not shown) for both baseline CDC group and previous antiretroviral therapy suggest that a proportional hazards assumption is not realistic for either variable.

Across strata formed by each combination of baseline CDC group and previous antiretroviral therapy, a stable partnership was associated with a slower rate of progression to new CDC group $\mathrm{C}$ disease or death (hazard ratio $0.79,95 \%$ confidence interval 0.63 to 0.98 ) (table 3). Exclusion of probable immune restoration diseases did not alter the result (hazard ratio 0.77 , 0.61 to 0.97 ). We found no evidence of an interaction between stable partnership and either baseline CDC group (difference in $\log$ likelihood ratio $0.9, \mathrm{df}=2, \mathrm{P}=0.64$ ) or previous antiretroviral therapy $(0.1, \quad \mathrm{df}=1, \mathrm{P}=0.74)$. This suggests that stable 


\begin{tabular}{|c|c|c|}
\hline Predictor & Adjusted hazards ratio (95\% CI) & $P$ value* \\
\hline Viral load (per $\log _{10}$ copies/ml) & $1.30(1.16$ to 1.45$)$ & $<0.01$ \\
\hline CD4 cell counts (per 100 cells/ $\mu \mathrm{l}$ ) & 0.85 (0.79 to 0.92$)$ & $<0.01$ \\
\hline Age (per 10 years) & 1.18 (1.05 to 1.32$)$ & 0.01 \\
\hline \multicolumn{3}{|l|}{ Sex: } \\
\hline Female & 1.19 (0.93 to 1.51$)$ & \multirow{2}{*}{0.17} \\
\hline Male & 1.00 (reference) & \\
\hline \multicolumn{3}{|l|}{ Transmission group: } \\
\hline Homosexual & 1.19 (0.90 to 1.57$)$ & \multirow{3}{*}{$<0.01$} \\
\hline Intravenous drug user & 1.92 (1.49 to 2.47$)$ & \\
\hline Heterosexual, otherf, or unknown & 1.00 (reference) & \\
\hline \multicolumn{3}{|l|}{ Education: } \\
\hline Higher & 0.94 (0.76 to 1.16$)$ & \multirow{2}{*}{0.57} \\
\hline Basic or unknown & 1.00 (reference) & \\
\hline \multicolumn{3}{|l|}{ Reported stable partnership: } \\
\hline Yes & 0.79 (0.63 to 0.98$)$ & \multirow{2}{*}{0.04} \\
\hline No & 1.00 (reference) & \\
\hline
\end{tabular}

*Difference in log likelihood ratio.

tTransmission through blood products or perinatal transmission.

partnership had a similar association with disease progression in each stratum. Nor did we find any evidence that a stable partnership had a different association if it first occurred after April 2000 (difference in $\log$ likelihood ratio $<0.1, \mathrm{df}=1, \mathrm{P}=0.94$ ), and our estimate of this association did not change when we included in our definition those reporting a stable partner without sexual intercourse. This indicates that the change in the way questions were asked did not materially influence our estimates of the association between stable partnership and disease progression.

Estimates for covariates (table 3) show that the rate of progression to new CDC group $\mathrm{C}$ disease or death increased with increasing viral load at baseline, with increasing age, and among intravenous drug users but decreased with increasing
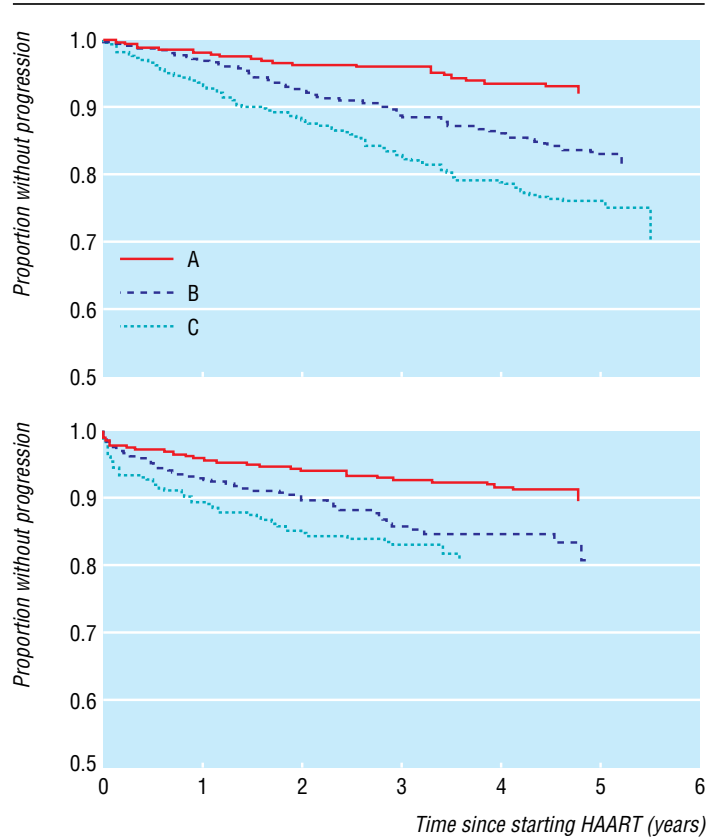

Fig 2 Proportion of participants without new CDC group C disease or death over time after starting highly active antiretroviral therapy (HAART). Kaplan-Meier curves for each clinical stage $(A, B$, and $C)$ at baseline for participants with previous antiretroviral therapy (top) and for those without previous antiretroviral therapy (bottom)
CD4 count at baseline. We found no evidence that the association between disease progression and stable partnership was any different for intravenous drug users (difference in log likelihood ratio $0.49, \mathrm{df}=1, \mathrm{P}=0.48)$.

Of 3309 patients who did not experience new CDC group C disease or death, $2896(88 \%)$ attended a follow up during the 12 months before 28 February 2002. We found no evidence of a "walking well" phenomenon, whereby patients with less severe disease were less likely to attend follow up. Compared with participants who had attended a recent follow up, those who had not were at a similar clinical stage at baseline, with similar baseline viral load and CD4 cell count (table 1).

\section{Secondary endpoints}

Stable partnership was associated with a decrease in the rate of progression to death and an increase in the rate of progression to CD4 cell counts of 50 and 100 above those recorded at baseline (table 4). We also found weaker evidence that stable partnership was associated with both an increase in the rate of progression to optimal viral suppression (hazard ratio 1.06, 0.98 to 1.14) and, in those achieving optimal suppression, with a decrease in the rate of progression to viral rebound (hazard ratio $0.91,0.80$ to 1.04$)$.

\section{Discussion}

Our analysis shows that a stable partnership is associated with a slower rate of disease progression in HIV infected patients receiving highly active antiretroviral therapy. The evidence for this is consistent across a range of surrogate marker and clinical endpoints. Our "intention to treat" definition of stable partnership was designed to avoid a liberal bias but may underestimate the strength of the association between stable partnership and disease progression. If, as figure 1d suggests, sicker patients are no more likely to discontinue stable partnerships because of the severity of their disease, then we could represent stable partnership as a variable that switches on and off as partnerships start and end. With this "as treated" definition, stable partnership is associated with a far greater decline in the rate of progression to new CDC group C disease or death (hazard ratio $0.67,95 \%$ confidence interval 0.55 to 0.81 ).

Our analysis has several possible limitations. Firstly, exclusions and censoring could lead to bias. Bias through informative censoring is unlikely, because $88 \%$ of those censored 
Table 4 Adjusted hazard ratios for the association between stable partnership and each endpoint

\begin{tabular}{|c|c|c|}
\hline Endpoint & Adjusted hazards ratio* $(95 \% \mathrm{Cl})$ & P value \\
\hline New CDC group, stage $C$ disease, or death & 0.79 (0.63 to 0.98$)$ & 0.04 \\
\hline Death & 0.59 (0.44 to 0.79$)$ & $<0.01$ \\
\hline CD4 increase by 50 & $1.11(1.03$ to 1.20$)$ & 0.01 \\
\hline CD4 increase by 100 & 1.15 (1.06 to 1.24$)$ & $<0.01$ \\
\hline Optimal viral suppression & $1.06(0.98$ to 1.14$)$ & 0.16 \\
\hline Viral rebound & $0.91(0.80$ to 1.04$)$ & 0.17 \\
\hline
\end{tabular}

${ }^{*}$ Cox proportional hazards model: strata—baseline clinical stage and previous antiretroviral therapy; covariates—viral load and CD4 cell count at baseline, age, sex, transmission group, and education.

†Difference in log likelihood ratio.

had been seen in the previous year. However, we excluded $30 \%$ of participants in the Swiss HIV cohort study who were taking HAART from our analysis, mostly because of missing baseline data. Predicting the direction of any bias is difficult, but clearly bias is possible.

Secondly, although we adjusted for the severity of the disease at baseline, a patient's health changes over time. "Time dependent confounding" occurs if sicker patients are less likely to form stable partnerships and these people are sicker because they were unable to form stable partnerships in the past. ${ }^{21}$ The effect of stable partnership is then confounded with disease severity. Many other factors-for example, mental health, drug adherence, and treatment efficacy-are potentially confounded with stable partnership in this way. Establishing a causal relationship with observational data is difficult. We have shown that after adjustment for baseline covariates an association remains between disease progression and stable partnership. The absence of a stable partnership is therefore a marker for an increased rate of disease progression, but that does not mean that the absence causes the increase.

Thirdly, the presence or absence of a stable partnership is an important but incomplete measure of the many ways in which social relationships influence health. Our definition of a stable partnership is likely to reflect received emotional support. One study to examine various aspects of social support found that greater emotional support was associated with a lower mortality in elderly people but that greater tangible support and the presence of a partner were not. ${ }^{8}$ However, the presence of a partner may be more important for younger adults. ${ }^{1}$

We can only speculate about the reasons why a stable partnership is associated with a slower rate of disease progression for people with HIV. The increased rate of progression to a CD4 cell increase and to viral suppression in patients with stable partners may be linked to drug adherence. ${ }^{22}$ People with a stable partner may have less depression, ${ }^{23}{ }^{24}$ a risk factor in many other chronic diseases. Research is needed on the mechanisms through which social support could influence HIV infection and should focus on mechanisms that are amenable to intervention. Increased support from healthcare givers might improve outcome in HIV infected people, but the evidence from one randomised trial in chronic cardiac patients does not support this hypothesis. ${ }^{25}$

\section{Conclusion}

The presence of a stable partnership is associated with a slower rate of disease progression in HIV infected patients who receive HAART, but the reasons for this are unknown. For a healthcare professional caring for someone with HIV, the absence of a stable partnership indicates that this patient may progress more rapidly through clinical latency to the later stages of the disease.

Swiss HIV Cohort Study Members: M Battegay, E Bernasconi, H C Bucher, P Bürgisser, M Egger, P Erb, W Fierz, M Fischer, M Flepp, P Francioli (presi-

\section{What is already known on this topic}

Social support has been associated with lower mortality among elderly people and people with cardiovascular disease

For people with HIV infection, social support has been linked to a slower decline in CD4 cell count, better adherence to treatment, and reduced risk behaviour

Little is known about the effect of social relationships on outcome in HIV infection

\section{What this study adds}

A stable partnership is associated with a slower rate of progression to AIDS or death in patients with HIV receiving highly active antiretroviral therapy

In the absence of a stable partnership an HIV infected patient may progress more rapidly through clinical latency to the later stages of the disease

dent of the SHCS, Centre Hospitalier Universitaire Vaudois, CH-1011Lausanne), H Furrer (chairman of the clinical and laboratory committee), M Gorgievski, H Günthard, P Grob, B Hirschel, L Kaiser, C Kind, T Klimkait, B Ledergerber, U Lauper, M Opravil, F Paccaud, G Pantaleo, L Perrin, J Piffaretti, M Rickenbach (head of data centre), C Rudin (chairman of mother and child substudy), J Schupbach, R Speck, A Telenti, A Trkola, P Vernazza (chairman of the scientific board), T Wagels, R Weber, S Yerly.

Contributors: HCB, RS, SDG, and JY designed the study. JY did the statistical analysis. MR, PV, MF, HF, EB, MB, BH, AT, and RW were responsible for patient recruitment, clinical assessment, and development of the questionnaire. MR was responsible for data quality. HCB, SDG, RS, and JY wrote the final report, assisted by all coauthors. HCB is the guarantor.

Funding: This study was financed in the framework of the Swiss HIV cohort study, supported by the Swiss National Science Foundation (Grant no 3345-062041). The Basel Institute for Clinical Epidemiology is supported by a grant from santésuisse and the Gottfried and Julia Bangerter-RhynerFoundation. HCB and JY received research grants from santésuisse and the Gottfried and Julia Bangerter-Rhyner-Foundation.

Competing interests: None declared.

Ethical approval: The Swiss HIV cohort study was approved by the local ethical committees of the participating centres.

1 Seeman TE, Kaplan GA, Knudsen L, Cohen R, Guralnik J. Social network ties and mortality among the elderly in the Alameda County study. Am J Epidemiol 1987;126:714-23.

2 Hanson BS, Isacsson SO, Janzon L, Lindell SE. Social network and social support influence mortality in elderly men: the prospective population study of "men born in 1914", Malmo, Sweden. Am J Epidemiol 1989;130:100-11.

3 Berkman LF, Leo-Summers L, Horwitz RI. Emotional support and survival after myocardial infarction: a prospective, population-based study of the elderly. Ann Intern Med 1992;117:1003-9.

4 Eng PM, Rimm EB, Fitzmaurice G, Kawachi I. Social ties and change in social ties in Eng PM, Rimm EB, Fitzmaurice G, Kawachi. Social ties and change in social ties in
relation to subsequent total and cause-specific mortality and coronary heart disease relation to subsequent total and cause-specific mo
incidence in men. Am J Epidemiol 2002;155:700-9.

5 Case RB, Moss AJ, Case N, McDermott M, Eberly S. Living alone after myocardial infarction: impact on prognosis. JAMA 1992;267:515-9. 
6 Williams RB, Barefoot JC, Califf RM, Haney TL, Saunders WB, Pryor DB, et al Prognostic importance of social and economic resources among medically treated patients with angiographically documented coronary artery disease. JAMA $1992 ; 267: 520-4$

7 Berkman LF, Glass T, Brissette I, Seeman TE. From social integration to health: Durkheim in the new millennium. Soc Sci Med 2000;51:843-57.

8 Penninx BW, van Tilburg T, Kriegsman DM, Deeg DJ, Boeke AJ, van Eijk JT. Effects of social support and personal coping resources on mortality in older age: the longitudinal aging study Amsterdam. Am J Epidemiol 1997;146:510-9.

9 Berkman LF. The role of social relations in health promotion. Psychosom Med 1995;57:245-54.

10 Uchino BN, Cacioppo JT, Kiecolt-Glaser JK. The relationship between social support and physiological processes: a review with emphasis on underlying mechanisms and implications for health. Psychol Bull 1996;119:488-531.

11 Leserman J, Petitto JM, Golden RN, Gaynes BN, Gu H, Perkins DO, et al. Impact of stressful life events, depression, social support, coping, and cortisol on progression to AIDS. Am J Psychiatry 2000;157:1221-8.

12 Theorell T, Blomkvist V, Jonsson H, Schulman S, Berntorp E, Stigendal L. Social support and the development of immune function in human immunodeficiency virus infection. Psychosom Med 1995;57:32-6.

13 Ingram KM, Jones DA, Fass RJ, Neidig JL, Song YS. Social support and unsupportive social interactions: their association with depression among people living with HIV. AIDS Care 1999;11:313-29.

14 Kimberly JA, Serovich JM. The role of family and friend social support in reducing risk behaviors among HIV-positive gav men. AIDS Educ Prev 1999;11:465-75.

15 Gordillo V, del Amo J, Soriano V, Gonzalez-Lahoz J. Sociodemographic and psychological variables influencing adherence to antiretroviral therapy. AIDS 999;13:1763-9.

16 Berkman LF, Syme SL. Social networks, host resistance, and mortality: a nine-year follow-up study of Alameda county residents. Am J Epidemiol 1979;109:186-204.

17 Levine C. AIDS and changing concepts of family. Milbank $Q$ 1990;68(suppl 1):33-58.

18 Ancelle-Park R. Expanded European AIDS case definition. Lancet 1993;341:441,

19 Glesby MJ, Hoover DR. Survivor treatment selection bias in observational studies: examples from the AIDS literature. Ann Intern Med 1996;124:999-1005.

20 Ledergerber B, Egger M, Erard V, Weber R, Hirschel B, Furrer H, et al. AIDS-related opportunistic illnesses occurring after initiation of potent antiretroviral therapy: the Swiss HIV cohort study. JAMA 1999;282:2220-6.

21 Robins JM, Hernan MA, Brumback B. Marginal structural models and causal inference in epidemiology. Epidemiology 2000;11:550-60.

22 Spire B, Duran S, Souville M, Leport C, Raffi F, Moatti JP. Adherence to highly active antiretroviral therapies (HAART) in HIV-infected patients: from a predictive to a dynamic approach. Soc Sci Med 2002;54:1481-96.

23 Page-Shafer K, Delorenze GN, Satariano WA, Winkelstein W, Jr. Comorbidity and survival in HIV-infected men in the San Francisco men's health survey. Ann Epidemio 1996;6:420-30.
24 Johnson JG, Alloy LB, Panzarella C, Metalsky GI, Rabkin JG, Williams JB, et al. Hopeessness as a mediator of the association between social support and depressive symptoms: findings of a study of men with HIV.J Consult Clin Psychol 2001;69:1056-60

25 Frasure-Smith N, Lesperance F, Prince RH, Verrier P, Garber RA, Juneau M, et al. Randomised trial of home-based psychosocial nursing intervention for patients recovering from myocardial infarction. Lancet 1997;350:473-9.

(Accepted 10 October 2003)

bmj.com 2004;328:15

Basel Institute for Clinical Epidemiology, University Hospital Basel, Hebelstrasse 10, Basle, CH-4031, Switzerland

Jim Young biostatistician

Heiner C Bucher professor of clinical epidemiology

Division of infectious Diseases and Hospital Hygiene, University Hospital Basel

Manuel Battegay professor of infectious diseases

Institute of Nursing Science, University of Basel, Basle

Sabina De Geest professor of nursing

Rebecca Spirig senior research fellow

Division of Infectious Diseases, University of Zurich, Zurich, Switzerland

Markus Flepp reader in internal medicine

Data Centre of the Swiss HIV Cohort Study, University of Lausanne, Lausanne, Switzerland

Martin Rickenbach epidemiologist

Division of Infectious Diseases, University Hospital Berne, Berne, Switzerland

Hansjakob Furrer reader in internal medicine

Ambulattorio malattie infettive, Ospedale Civico, Lugano, Switzerland

Enos Bernasconi head of unit

Division des maladies infectieuses, Hôpital Universitaire de Genève, Geneva,

Switzerland

Bernard Hirschel professor of infectious diseases

Division des maladies infectieuses, Centre Hospitalier Universitaire Vaudois,

Lausanne

Amalio Telenti professor of infectious diseases

Division of Internal Medicine, Cantonal Hospital Saint Gall, Saint Gall, Switzerland Pietro Vernazza reader in internal medicine

Correspondence to: $\mathrm{H}$ C Bucher

hbucher@uhbs.ch 